\title{
A novel surgical approach to left main coronary artery giant aneurysm thrombosis in a child with a history of Kawasaki disease
}

\author{
Jay D. Pruetz, MD, ${ }^{\mathrm{a}, \mathrm{c}}$ Masato Takahashi, MD, ${ }^{\mathrm{a}, \mathrm{c}}$ Brian L. Reemtsen, MD, ${ }^{\mathrm{a}, \mathrm{b}}$ and Vaughn A. Starnes, MD, ${ }^{\mathrm{a}, \mathrm{b}}$ Los Angeles, California
}

A giant aneurysm involving the left main coronary artery (LMCA) is a life-threatening complication of Kawasaki disease. Systemic anticoagulation is the mainstay of medical therapy for this complication. Once thrombosis occurs, thrombolytic therapy is usually attempted. If thrombolysis fails to reopen the lumen, an invasive procedure is needed to preserve myocardial perfusion. Previous approaches have used coronary artery bypass grafting, interposition grafting, and transcatheter revascularization. We describe a novel surgical approach to a thrombosed giant aneurysm in the LMCA.

\section{CLINICAL SUMMARY}

Kawasaki disease was diagnosed in a 5-year-old boy after he demonstrated persistent fever post-appendectomy. His initial therapy produced only temporary defervescence, and a giant saccular aneurysm of the LMCA eventually developed. Despite systemic anticoagulation, the patient had an acute myocardial infarction 9 months later because of a thrombus in the LMCA aneurysm. He recovered from myocardial infarction after treatment with thrombolytic therapy but continued to have chronic, subclinical thrombus for-

From the Childrens Hospital Los Angeles, ${ }^{\mathrm{a}}$ Los Angeles, California, and Departments of Cardiothoracic Surgery ${ }^{\mathrm{b}}$ and Pediatrics, ${ }^{\mathrm{c}}$ University of Southern California, Keck School of Medicine, Los Angeles, California.

Received for publication Feb 6, 2008; accepted for publication Feb 17, 2008.

Address for reprints: Jay D. Pruetz, MD, Childrens Hospital Los Angeles, Department of Pediatrics, 4650 Sunset Blvd, Los Angeles, CA 90027 (E-mail: jpruetz@chla.usc.edu). J Thorac Cardiovasc Surg 2009; 137:1030-2

$0022-5223 / \$ 36.00$

Copyright (c 2009 by The American Association for Thoracic Surgery doi:10.1016/j.jtcvs.2008.02.048 mation. At age 9 years, a cardiac catheterization showed a large thrombus within the giant LMCA aneurysm partially obstructing his left anterior descending (LAD) artery and completely occluding his circumflex artery (Figure 1). He was also noted to have unusually small internal thoracic arteries. The patient underwent a novel surgical repair that involved opening the main pulmonary artery transversely to gain better exposure to the aneurysm. The aneurysmal sac was dissected out and then incised to remove the thrombus. The posterior walls of the native LMCA and LAD were identified within the giant aneurysm, forming a continuous endothelial tract from the left coronary ostium to the LAD. A ring of pulmonary artery tissue was excised and used to reroof the endothelial tract within the aneurysm, creating a new LMCA and excluding the rest of the giant aneurysm (Figure 2). The patient tolerated the surgery well with no postoperative complications. Since the patient's operation, 24 months ago, he has done well on low-dose aspirin therapy with no subsequent hospitalizations or interventions. Recent cardiac catheterization showed a widely patent tract from the LMCA to the LAD with no recurrence of thrombus (Figure 1). Follow-up nuclear cardiac scan and magnetic resonance imaging and angiography showed no perfusion defects or wall motion abnormalities with normal left ventricular function. Now at age 11 years, the patient remains completely asymptomatic and active.

\section{DISCUSSION}

Surgical and transcatheter revascularization techniques have been used successfully for patients with high-grade obstruction in at least 2 major coronary arteries or in the LMCA
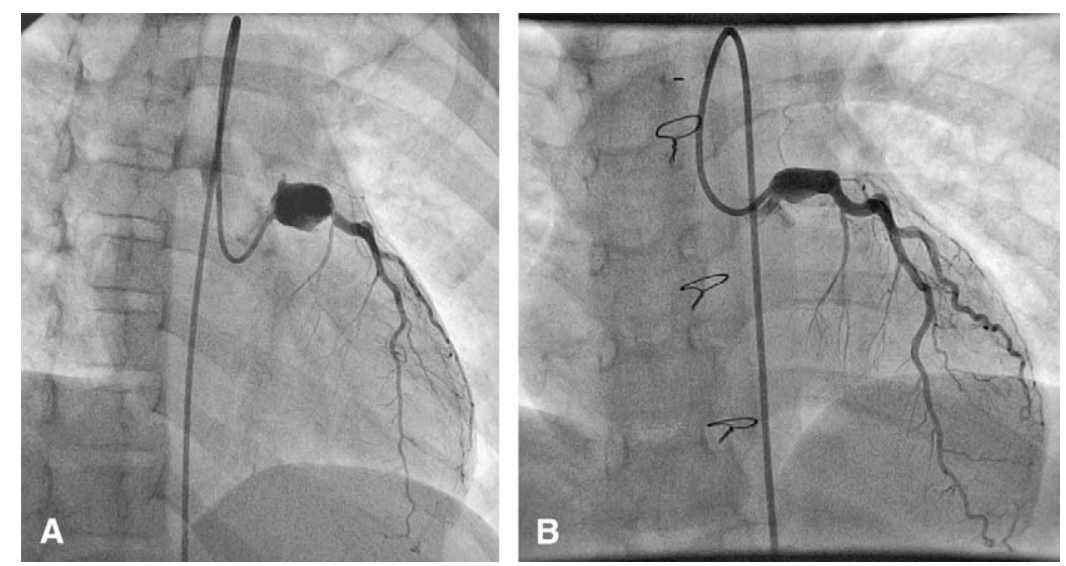

FIGURE 1. A, Preoperative cardiac angiogram of a giant LMCA aneurysm with thrombus causing a filling defect of the proximal LAD and complete occlusion of the circumflex artery. B, Postoperative angiogram of the LMCA at 24 months showing widely patent repair that fills the LAD and septal perforating branches. The circumflex is fed entirely by collateral blood flow from the RCA (not seen on this injection). $L A D$, Left anterior descending; $L M C A$, left main coronary artery. 

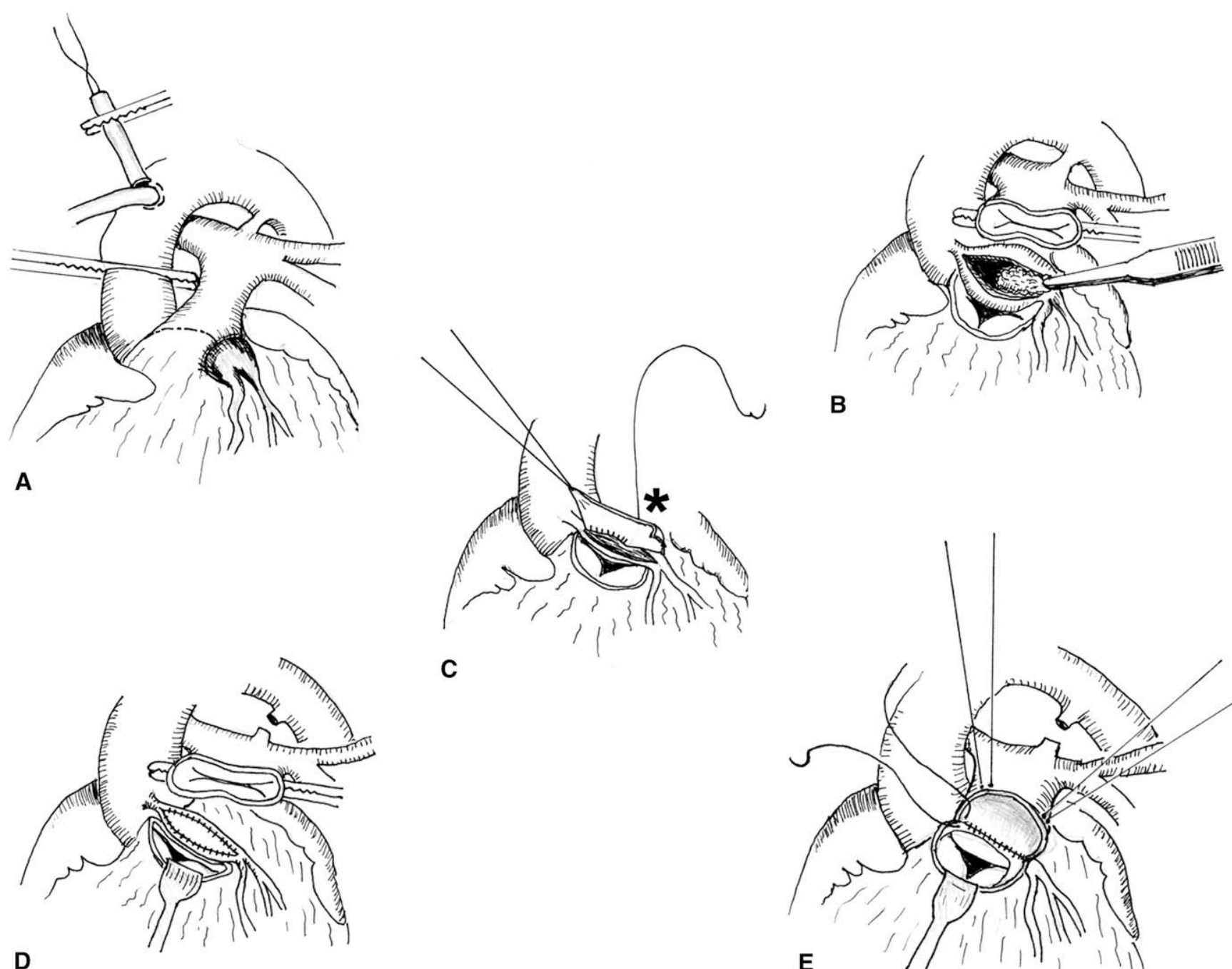

C

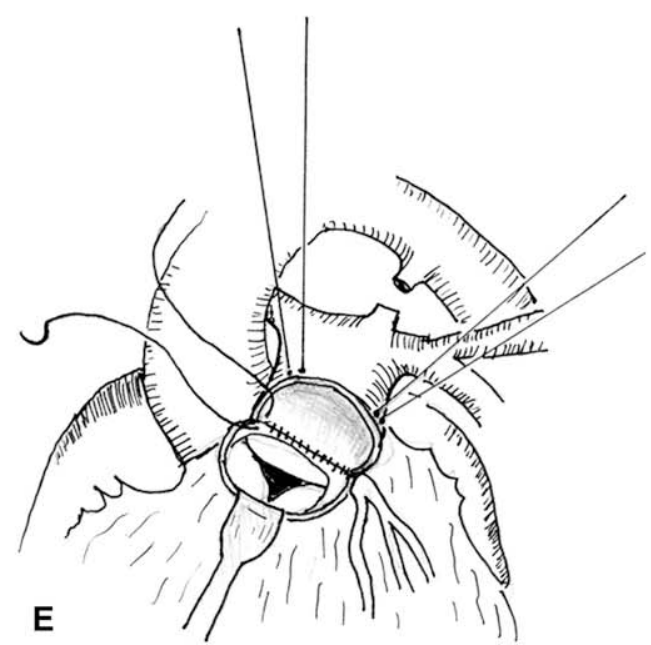

FIGURE 2. Diagram for novel surgical repair of a giant LMCA aneurysm with occlusive thrombus. A, LMCA aneurysm was readily noticeable on the anterior surface of the heart just lateral to the main pulmonary artery. B, Main pulmonary artery was opened transversely over the aneurysmal portion of the LMCA, and the aneurysmal sac was dissected out. Unroofing and resection of giant LMCA aneurysm were performed with removal of thrombus. A continuous endothelial tract was identified from the left main ostium to the LAD. C, Ring of main pulmonary artery was excised and opened for use as patch material $(*)$. Reroofing of the endothelial tract from the LCA ostium to the LAD was performed using the autologous tissue and a running stitch. $\mathrm{D}$, Completed repair of LMCA. The ligamentum arteriosum was divided to mobilize the main pulmonary artery segment for end-to-end repair. E, Repair is completed by end-to-end anastomosis of the transected main pulmonary artery. $L A D$, Left anterior descending; $L C A$, left coronary artery; $L M C A$, left main coronary artery; $M P A$, main pulmonary artery.

because of the elevated risk of fatal myocardial infarction. Current surgical approaches to giant coronary aneurysms with thrombosis often use coronary artery bypass graft techniques with either internal thoracic or gastroepiploic arteries to provide arterial blood flow distal to the aneurysm with or without ligation of the aneurysm. These techniques have generally resulted in good 10-year patency rates if adequate graft flow is confirmed by 1-year postbypass. However, patients aged less than 12 years tend to have lower graft patency rates and may require percutaneous transluminal balloon angioplasty in the first few months to prevent graft occlusion. ${ }^{1}$ Several reports have also described the use of in- terposition grafts using a saphenous vein or circumflex femoral artery. ${ }^{2,3}$ Although this alternative method preserves the normal coronary flow pattern, it may not be a viable option in a child with smaller caliber blood vessels and may lead to greater postoperative disability and recovery time because of the second surgical site. Transcatheter interventions, such as percutaneous transluminal balloon angioplasty, percutaneous transluminal coronary rotational ablation, and coronary stent placement, have produced good short-term outcomes. However, their long-term efficacy remains unclear because of low 5-year patency rates and high incidence of neoaneurysms at intervention sites. ${ }^{4}$ Another surgical approach 
previously described for giant right coronary artery (RCA) aneurysms involved the excision of the RCA aneurysm with mobilization of the RCA inflow and outflow portions, followed by end-to-end anastomosis. ${ }^{5}$ This repair preserved the native vessel continuity and did not require lifelong anticoagulation therapy. However, end-to-end anastomosis runs the risk of suture line tension causing restenosis and may not be technically feasible for patients with large aneurysms.

\section{CONCLUSIONS}

Our surgical approach to thrombosis of a giant LMCA aneurysm has the primary advantage of using native host tissue to maintain a more anatomically normal continuity from the aorta to the coronary artery without the use of an interposition or bypass graft. Furthermore, it avoids the potential for myocardial ischemia resulting from compromised proximal perforating branch flow that may occur with bypass and liga- tion of a coronary aneurysm or percutaneous covered stent techniques. Our novel surgical approach also obviates the need for lifelong anticoagulant therapy.

\section{References}

1. Tsuda E, Kitamura S, Kimura K, Kobayashi J, Miyazaki S, Echigo S, et al. Longterm patency of internal thoracic artery grafts for coronary artery stenosis due to Kawasaki disease: comparison of early with recent results in small children. Am Heart J. 2007; 153:995-1000.

2. Firstenberg MS, Azoury F, Lytle B, Thomas JD. Interposition vein graft for giant coronary aneurysm repair. Ann Thorac Surg. 2000;70:1397-8.

3. Anderson CA, Filsoufi F, Kadner A, Adams DH. Repair of a left main coronary artery aneurysm using the circumflex femoral artery as a Y-interposition graft. Ann Thorac Surg. 2004;78:314-6.

4. Ishii M, Ueno T, Ikeda H, Iemura M, Sugimura T, Furui J, et al. Sequential followup results of catheter intervention for coronary artery lesions after Kawasaki disease: quantitative coronary artery angiography and intravascular ultrasound imaging study. Circulation. 2002;105:3004-10.

5. Westaby S, Vaccari G, Katsumata T. Direct repair of giant right coronary aneurysm. Ann Thorac Surg. 1999;68:1401-3.

\title{
Resection and reconstruction of the roof of the coronary sinus for an unusually attached left atrial myxoma
}

\author{
Igor E. Konstantinov, MD, PhD, Pankaj Saxena, MCh, DNB, Trenton Barrett, MB, BS, and Graham Jenkins, BPHE, Perth, Australia
}

Myxoma is the most common primary cardiac tumor and most commonly originates from the left side of the atrial septum. We encountered an unusual myxoma with a broad pedicle that originated from the lower atrial septum and the roof of the coronary sinus (CS), causing mild mitral stenosis.

\section{CLINICAL SUMMARY}

A 69-year-old woman was admitted to the Sir Charles Gairdner Hospital with acute shortness of breath. On the patient's admission, a transient ischemic attack developed with left arm weakness that spontaneously resolved. She had a long-standing history of type II diabetes mellitus, hypertension, hypercholesterolemia, and paroxysmal atrial fibrillation. Despite an appropriate anticoagulation with

\footnotetext{
From the Department of Cardiothoracic Surgery, Sir Charles Gairdner Hospital, University of Western Australia, Perth, Australia.

Received for publication Jan 26, 2008; accepted for publication Feb 10, 2008.

Address for reprints: Igor E. Konstantinov, MD, PhD, University of Western Australia, Department of Cardiothoracic Surgery, Sir Charles Gairdner Hospital, Hospital Avenue, Nedlands, Perth, WA 6009, Australia (E-mail: konstantinov.igor@alumni. mayo.edu).

J Thorac Cardiovasc Surg 2009; 137:1032-4

0022-5223/\$36.00

Copyright (c) 2009 by The American Association for Thoracic Surgery doi:10.1016/j.jtcvs.2008.02.047
}

warfarin, she had multiple transient ischemic attacks in the past. Coronary angiogram was normal. A transthoracic echocardiography revealed a $5.4 \times 3.4-\mathrm{cm}$ pedunculated left atrial mass that was attached to the lower atrial septum (Figure 1). The mass was prolapsing into the inlet of mitral valve during diastole (Figure 1, $A$ ) and moving back into the left atrium in systole (Figure 1,B). Left ventricular function was normal, and there was no significant mitral regurgitation. Urgent surgery was performed. After standard median sternotomy and initiation of cardiopulmonary bypass, antegrade cold cardioplegia was administered, and a biatrial approach by means of an inverted T-shaped incision (Figure 2, $A$ ) was used to expose the mass, which had a typical appearance of a myxoma. The incision was extended across the septum and around the broad attachment of the tumor (Figure $2, B)$. An unusual feature of the myxoma was its broad attachment to the lower septum and the roof of the CS (Figure 2, $C$ and $D$ ). The roof of the CS was resected to completely remove the attachment site of the myxoma (Figure 2, $D$ and $E$ ). The roof of the CS and the atrial septal defect were reconstructed with a double-layered autologous pericardial patch, and the atriotomy was closed in standard fashion (Figure 2, $F, G$, and $H$ ). No other intracardiac abnormalities were found. Aortic crossclamp time was 33 